\title{
Clinical Research on the Method of Huafu Zaisheng in Treating Diabetic Foot with Tendons Necrotic*
}

\author{
Chaojun Zhu, Jing Ma, Xianzhou Liu, Xuya Lu, Qiang Xu, Zhaohui Zhang\# \\ Second Affiliated Hospital of Tianjin University of TCM, Tianjin, China \\ Email:"zzh45@aliyun.com
}

Received 18 January 2016; accepted 1 February 2016; published 5 February 2016

Copyright (C) 2016 by authors and OALib.

This work is licensed under the Creative Commons Attribution International License (CC BY). http://creativecommons.org/licenses/by/4.0/

cC) (i) Open Access

\section{Abstract}

Objective: To observe the effect of liquefying the necrotic tendons of diabetic foot and promotion of wound healing using the treatment of Huafu Zaisheng. Methods: 98 patients suffering from diabetic foot were randomly divided into Huafu Zaisheng group (35 cases), Huafu group (32 cases), Zaisheng group (31 cases), and then had eroded debridement timely for its wounds. Since then, different methods and drugs applied to deal with the wound, observe the liquefaction time and detachment time of the degeneration and necrosis tendon, and observe the wound healing. Collection and preservation of wound secretion, determine the $\mathrm{pH}$ value of wound secretion, determination content of the NO and TGF- $\beta 1$ in wound secretion. Wound tissue containing fascia and tendons was extracted by surgical methods, before treatment and 6, 12 weeks after treatment. All tissue samples were prepared according to Hematoxylin-eosin staining techniques, after which their appearance was evaluated for pathological changes using an optical microscope. Immunohistochemistry staining was used to detect the expressions of CD34 and CK19. Results: By comparison of the efficacy of the three groups, the curative effect of the HFZS group is superior to HF and ZS group. The necrotic tendons of Huafu Zaisheng group were liquefied clearer than the other two groups. Three groups of secretions $\mathrm{pH}$ value were the trend of first decreased, then increased, weakly basic first, and then weak acid, and then close to neutral. Compared to ZS group and HF group, the difference was statistically significant $(P<0.01)$. After 4.6 weeks' treatment, compared to ZS group and HF group, the NO, TGF- $\beta 1$ content of HFZS group increased significantly, and the difference was statistically significant $(P<0.01)$. Conclusion: Using the method of Huafu Zaisheng, the necrotic tendons and tissues could be liquefied as early as possible, and then promote the wound healing. Application of Huafuzaisheng method to deal with wounds, can remove necrotic tendons and fascia tissue as early as possible, can change the wound $\mathrm{pH}$ value and provide a variety of active factors (NO, TGF- $\beta 1$ ) and micro-environment for wound healing.

\footnotetext{
*The priority special disease and speciality project of traditional Chinese medicine in Tianjin.

"Corresponding author.
}

How to cite this paper: Zhu, C.J., Ma, J., Liu, X.Z., Lu, X.Y., Xu, Q. and Zhang, Z.H. (2016) Clinical Research on the Method of Huafu Zaisheng in Treating Diabetic Foot with Tendons Necrotic. Open Access Library Journal, 3: e2388.

http://dx.doi.org/10.4236/oalib.1102388 


\title{
Keywords
}

\author{
Huafu Zaisheng, Diabetic Foot, Bromelain, Shengji Corium Elephatis Mastic, Tendons \\ Subject Areas: Diabetes \& Endocrinology, Infectious Diseases, Surgery \& Surgical Specialties
}

\section{Introduction}

Diabetic foot ulcers were typically chronic wounds which were difficult to heal. This was due to a range of pathogenic abnormalities in diabetics, which included is chaemia and intrinsic defects in angiogenesis and impaired immunity against infection [1]. Diabetic foot ulcers commonly become infected and lead to amputation. In Chinese society, preserving the foot is a major hope of diabetic patients and their families. Tendon infection and necrosis are difficult to handle for surgeon. Once the foot tendon undergoes degeneration, necrosis and ulcers occur and the need for amputation greatly increases. Currently, most doctors take of mechanical debridement to removal of necrotic tendon. However, it is difficult to define the scope of debridement, and the microcirculation around the wound is damaged by the mechanical debridement which increased tissue ischemia and gangrene. Improper debridement caused to the spread of infection. For these difficulties, we propose the method of removing necrotic tissue and promoting granulation (Huafu Zaisheng method). This method can be liquefied the tendon of necrosis, thereby promoting wound healing. We applied this method of treatment of 35 cases, and obtained satisfactory efficiency. These are as follows:

\section{Clinical Data}

\subsection{Study Population}

We present 98 cases of diabetic patients with foot ulcers wagner 3 - 4. These patients were treated from January 2009 to October 2015. They were divided into three groups randomly, there are Huafu Zaisheng group (abbreviated as HFZS group), Huafu group (abbreviated as HF group) and Zaisheng group (abbreviated as ZS group).

22 male and 13 female patients were included in HFZS group; the mean age of the patients was $66.56 \pm 11.62$ years (range, 32 - 79 years). The wounds were initially classified as grade 3 (20 cases), grade 4 (15 cases), according to the Wagner [2] classification of diabetic foot ulcers 20 male and 12 female patients were included in HF group; the mean age of the patients was $67.88 \pm 14.45$ years (range, $30-80$ years). The wounds were initially classified as grade 3 (17 cases), grade 4 (15 cases). 17 male and 14 female patients were included in ZS group; the mean age of the patients was $65.21 \pm 15.73$ years (range, 33 - 79 years). The wounds were initially classified as grade 3 (18 cases), grade 4 (13 cases).

These patients had poorly controlled diabetes, and their mean glycosylated hemoglobin (HbA1c) level was 7.5\%. Arterial Duplex scans were performed and the ankle-brachial index less than 0.5 , in most patients. No remarkable significant difference among the three groups, compared with the age, sex, Wagner classification, wound area, and the course of DM (see Table 1).

\subsection{Drugs and Equipment, Reagents}

Shengji corium elephatis mastic (approval number: jin medicine Z20070652). Huafuzaisheng powder: bromelain is the main component. NO and the TGF- $\beta 1$ ELISA kit, Rabbit Anti-Cytokeratin 19 antibody, Rabbit AntiCD34 antibody, Beijing Bioss Biotechnology Co., Ltd. pH precision test paper, MACHEREY-NAGEL, Germany.

Table 1. Patient demographics, Wagner classification, wound area, the course of DM.

\begin{tabular}{cccccccc}
\hline Group & N & Male & Female & Age (years) & Wagner 3 & Wagner 4 & Wound area $\left(\mathrm{cm}^{2}\right)$ \\
\hline HFZS & 35 & 22 & 13 & $66.56 \pm 11.62$ & 20 & 15 & $239.17 \pm 35.21$ \\
HF & 32 & 20 & 12 & $67.88 \pm 14.45$ & 17 & 15 & $245.34 \pm 38.15$ \\
ZS & 31 & 17 & 14 & $65.21 \pm 15.73$ & 18 & 13 & $236.36 \pm 39.23$ \\
\hline
\end{tabular}




\subsection{Diagnostic Criteria}

98 cases patients were enrolled with diabetic foot tendon exposure, necrosis, Grade 3 (ulcer with osteomyelitis or abscess), Grade 4 (gangrene to portion of forefoot) according to Wagner's Classification [2]. 30 to 80 years old.

\subsection{Inclusion Criteria and Exclusion Criteria}

Inclusion criteria: Patients with diabetic foot, Wagner class 3 - 5, any gender, ages 30 to 80 years.

Exclusion criteria: 1) Patients who can't complete this test as our required; 2) Patient's important organs has severe disease, such as the abnormal hepatic function, renal failure, heart failure, malignant tumor, etc.; 3) Pregnant woman, patients allergic to medicines.

\subsection{Ethical and Moral Issues}

This study was approved by The Second Affiliated Hospital Tianjin University of TCM ethics committee. All the patients were signed the informed consent.

\subsection{Evaluation Standard}

Clinical cure: the wound is completely healed; Significantly effective: necrotic tendons was liquefied and fall off, wound area reduced by more than two-thirds; effective: a part of necrotic tendons was liquefied and fall off, Wound area reduced between a quarter to two-thirds; Ineffective: Wound infection spread, necrosis of tendon increased, wound area reduced less than a quarter.

\subsection{Medical Management}

Control the level of blood glucose, reduce platelet aggregation rate, decrease blood lipid, controlling blood pressure. According to the results of wound secretion culture and drug susceptibility, choose sensitive antibiotic. With pus cavity formation in patients with diabetic foot, surgical incision and drainage. Diabetic foot patients presented with varying degrees of lower limb ischemia. For patients can choose lower limb vascular lumen treatment, if according with operation standard, actively improve circulation.

\subsection{Method of Removing Necrotic Tissue and Promoting Granulation Surgical Treatment}

Disinfect skin and wound regularly. These patients required immediate drainage and infection control. Remove the necrotic tissue or normal tissue where obstacles to drainage. The degeneration of tissue, it not hinder the drainage and the boundary is not clear, can delay to clear. HFZS group: surgical treatment use huafuzaisheng powder joint Shengji corium elephatis mastic. Huafuzaisheng powder sprinkled to necrosis and degeneration of tendon, the wound was covered Shengji corium elephatis mastic. HF group: Huafuzaisheng powder sprinkled to necrosis and degeneration of tendon, the wound was covered vaseline gauze. ZS group: The wound was covered with Shengji corium elephatis mastic, bandaging after surgical treatment. Area of Shengji corium elephatis mastic and vaseline gauze is less than the area of the wound, make sure that wound drainage unblocked, to prevent wound infection spread. Surgical treatment 1 times a day, observe 16 weeks.

\section{Indexes and Methods}

\subsection{Wound Observation}

Observe the time of diabetic foot necrosis tendon falls off. Observe the amount of wound secretion, granulation growth quality of wound, epithelial growth of the wound peripheral. We compared the cure rate and the effective rate among the three groups.

\subsection{Collection and Preservation of Wound Secretion}

Acquisition of wound secretion of every patient before treatment, collected wound secretion of every patient after 2 weeks, 4 weeks, 6 weeks treatment. The wound secretion of patients collected into $1.5 \mathrm{ml}$ polypropylene centrifugal tube, $-20^{\circ} \mathrm{C}$ refrigerator frozen $8 \mathrm{~h}$, put specimens in freeze drying machine, continuous boot $24 \mathrm{~h}$, the sample store in a refrigerator for $4^{\circ} \mathrm{C}$ refrigerated after lyophilization. At the time of measurement, using elec- 
tronic analytical balance according to take about $40 \mathrm{mg}$ of freeze-drying discharge, injection ten times of $0.9 \%$ NS, application of electronic ultrasonic homogenate, homogenate $30 \mathrm{~s}$, make it into slurry. Refrigerated centrifuge $12,000 \mathrm{RPM}, 4^{\circ} \mathrm{C}$ refrigerated centrifuge for $10 \mathrm{~min}$, absorb the supernatant fluid of standby as test specimen.

\subsection{Determine the pH Value of Wound Secretion}

Acquisition of wound secretion of every patient before treatment, collected wound secretion of every patient after 2 weeks, 4 weeks, 6 weeks treatment. $\mathrm{pH}$ meter is used to test the $\mathrm{pH}$ value of the wound secretion.

\subsection{Determination Content of the NO and TGF- $\beta 1$ in Wound Secretion}

Took the specimen from the refrigerator freezer, in the specimen, we tested the content of NO and TGF- $\beta 1$ respectively, applications the NO and the TGF- $\beta 1$ ELISA kit.

\subsection{Pathology Detection of the Wound}

Tendon and fascia necrosis tissue was taken before treatment. After 6 and 12 weeks treatment, degeneration tendons and granulation tissue, epithelium containing granulation tissue was taken respectively. HE staining, CD34 and CK19 immunohistochemical were done. Application of special apparatus used to get cylindrical the above materials, it's diameter is $0.5 \mathrm{~cm}, 0.3 \mathrm{~cm}$ high. Tissue specimens were fixed in $10 \%$ formalin solution, routinely paraffin embedding, sectioning. Slices were observed by optical microscope, after HE staining and immunohistochemical staining.

\subsection{Statistical Analysis}

SPSS 11.0 statistical software was used for single-factor analysis of variance and pair wise comparisons between groups. The enumeration data was compared by $\chi^{2}$ test. A $t$-test was used to compare between areas of wounds before and after treatment with three therapies. A $P$ value of 0.05 was used to determine statistical significance.

\section{Results}

Comparison of the efficacy of the three groups, the curative effect of the HFZS group is superior to HF and ZS group $(P<0.05$, Table 2$)$. Wound healing following the rule of removing necrotic tissue and promoting granulation, proper growth of granulation tissue promotes epithelium growth. When there is necrosis tendon and necrotic tissue, wound secretion increased, pus thin, stinking, even with blood. When the necrosis of tendon and organization gradually fall off, granulation tissue begins to grow, capillary invasion into the tendon, part of the degeneration of tendon reversal, granulation tissue wrap it gradually, promote again epithelial change and ulcer cicatrizations (Figures 1 (a)-(d)).

1) Comparison the secretions $\mathrm{pH}$ value of three groups

Observation shows that: in the treatment process, three groups of secretions $\mathrm{pH}$ value were the trend of first decreased, then increased, weakly basic first, and then weak acid, and then close to neutral. Before treatment, each group of secretions $\mathrm{pH}$ value are weak alkaline. After 2 weeks treatment, the $\mathrm{pH}$ value were falling, but no statistically significant difference among groups $(P>0.05)$. After 4 weeks treatment, the $\mathrm{pH}$ value achieved the minimum, compare to ZS group, the difference was statistically significant $(P<0.01)$. After 6 weeks treatment, the $\mathrm{pH}$ value began to rise, compare to ZS group and HF group, the difference was statistically significant $(P<$ 0.01, Table 3). See Figure 2.

Table 2. Comparison of the efficacy of the three groups (cases).

\begin{tabular}{cccccccc}
\hline Group & $n$ & Clinical cure & Significantly effective & Effective & Ineffective & Cure rate & Effective rate \\
\hline HFZS & 35 & 21 & 7 & 5 & 2 & $60^{\triangle \Delta}$ & $94.3^{\triangle \boldsymbol{\Delta}}$ \\
HF & 32 & 12 & 9 & 5 & 10 & 33 & 72.2 \\
ZS & 31 & 11 & 8 & 8 & 9 & 31 & 75.0 \\
\hline
\end{tabular}

Notes: compare to HF group, ${ }^{\triangle} P<0.05$; compare to ZS group, ${ }^{\wedge} P<0.05$. 


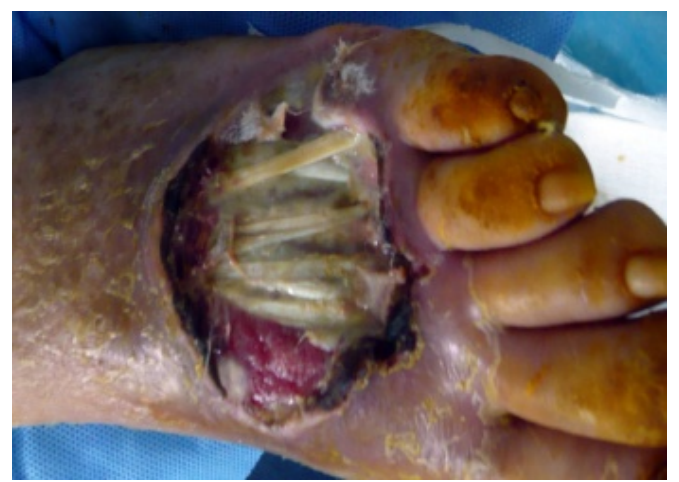

(a)

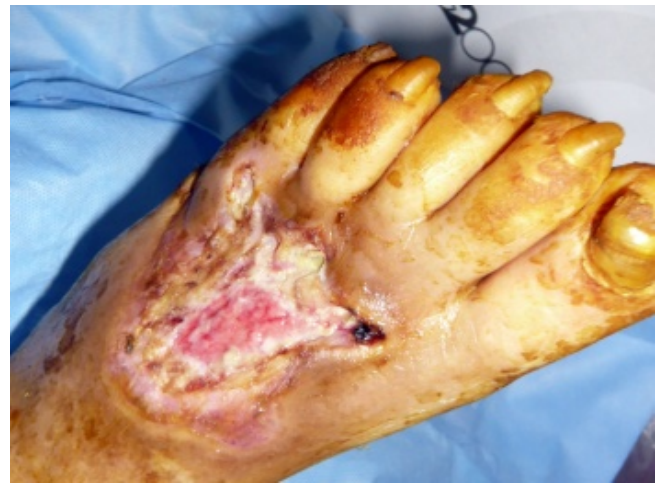

(c)

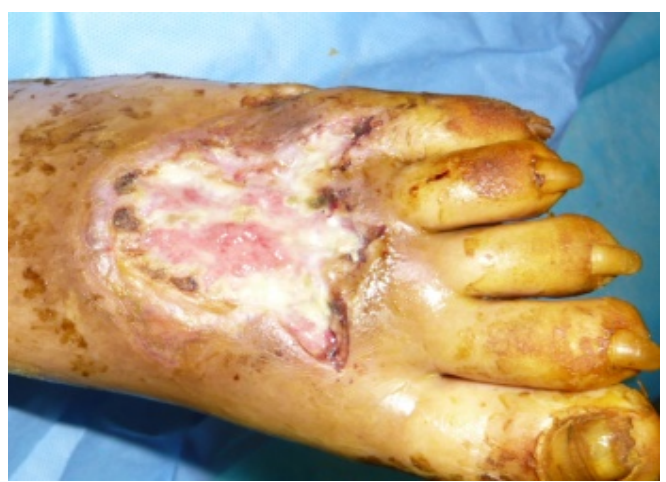

(b)

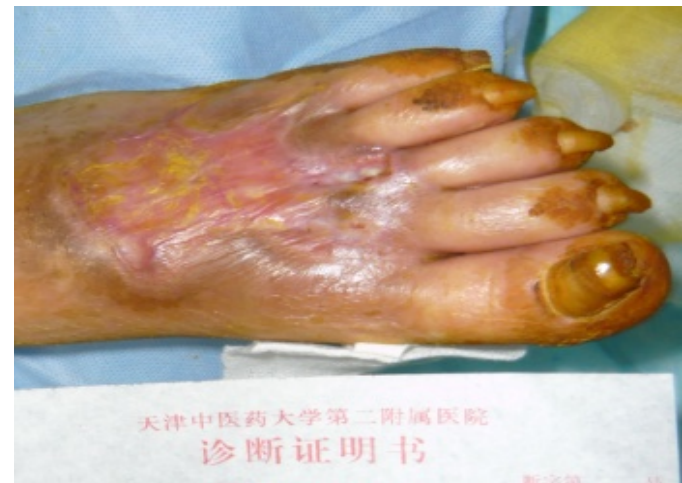

(d)

Figure 1. (a) Before treatment, we can see a large number of necrotic tissue and necrotic tendons; (b) After 8 weeks treatment, the necrosis of tendon and organization gradually fall off, granulation tissue begins to grow; (c) After 12 weeks treatment, capillary invasion into the tendon, granulation tissue wrap it gradually; (d) After 16 weeks treatment, epithelial change and ulcer cicatrizations.

\section{PH value}

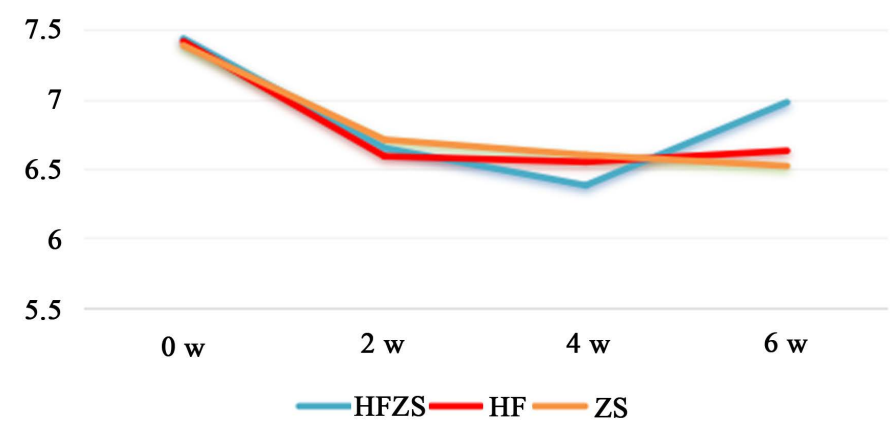

Figure 2. $\mathrm{pH}$ value of the three groups.

Table 3. The secretions $\mathrm{pH}$ value of three groups $(\bar{x} \pm s)$.

\begin{tabular}{|c|c|c|c|c|c|}
\hline Group & $n$ & $0 \mathrm{w}$ & $2 \mathrm{w}$ & $4 \mathrm{w}$ & $6 \mathrm{w}$ \\
\hline HFZS & 35 & $7.44 \pm 0.27$ & $6.65 \pm 0.41$ & $6.38 \pm 0.26$ & $6.98 \pm 0.20$ \\
\hline $\mathrm{HF}$ & 32 & $7.42 \pm 0.33$ & $6.59 \pm 0.36$ & $6.55 \pm 0.37$ & $6.63 \pm 0.27^{* *}$ \\
\hline $\mathrm{ZS}$ & 31 & $7.39 \pm 0.31$ & $6.71 \pm 0.31$ & $6.67 \pm 0.33^{\triangle \Delta}$ & $6.52 \pm 0.23^{\triangle \Delta}$ \\
\hline
\end{tabular}

Notes: compare to HF group, ${ }^{*} P<0.05$, ${ }^{* *} P<0.01$; compare to ZS group, ${ }^{\triangle} P<0.05,{ }^{\triangle \triangle} P<0.01$. 
2) Comparison the nitric oxide (NO) content in secretions among three groups

Observation shows that: in the treatment process, the amount of NO present a trend of increasing. After 4.6 weeks treatment, compare to ZS group and HF group, the NO content of HFZS group increased significantly, the difference was statistically significant $(P<0.01$, Table 4$)$. Compared between HF and ZS group, there was no statistically significant difference $(P>0.05)$. See Table 4 and Figure 3.

3) Comparison the TGF- $\beta 1$ content in secretions among three groups

In the treatment process, the amount of TGF- $\beta 1$ present a trend of increasing. After 2, 4, 6 weeks treatment, compare to ZS group and HF group, the TGF- $\beta 1$ content of HFZS group increased significantly, the difference was statistically significant $(P<0.01$, Table 5). Compared between HF and ZS group, there was no statistically significant difference $(P>0.05)$. See Table 5 and Figure 4 .

4) The pathological changes of HFZS method promote wound healing

The necrotic tissue liquefied and fell off period, there is a lot of necrotic tissue. Necrosis of tendon and fascia contains large amounts of inflammatory ooze and necrotic cells. When necrosis tendons and fascia gradually fall off, inflammatory ooze, necrotic material and inflammatory granulation tissue exist together. Vascular endothelial cells proliferation actively, closely packed and upright in the granulation tissue, a large number of neutrophilic ooze in the stroma, CD34 staining positive expression of capillary endothelial cells. Regeneration period, wound filled with granulation tissue, epithelial change gradually, clinical recovery (Figures 5(a)-(d)).

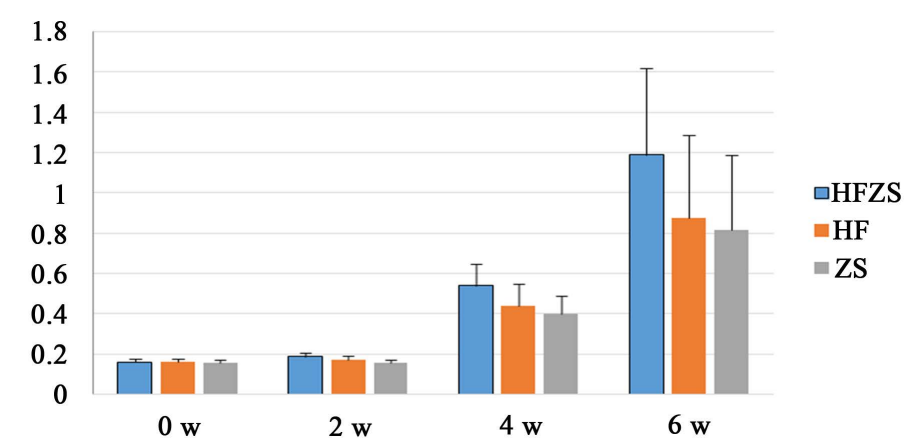

Figure 3. NO content in secretions among three groups.

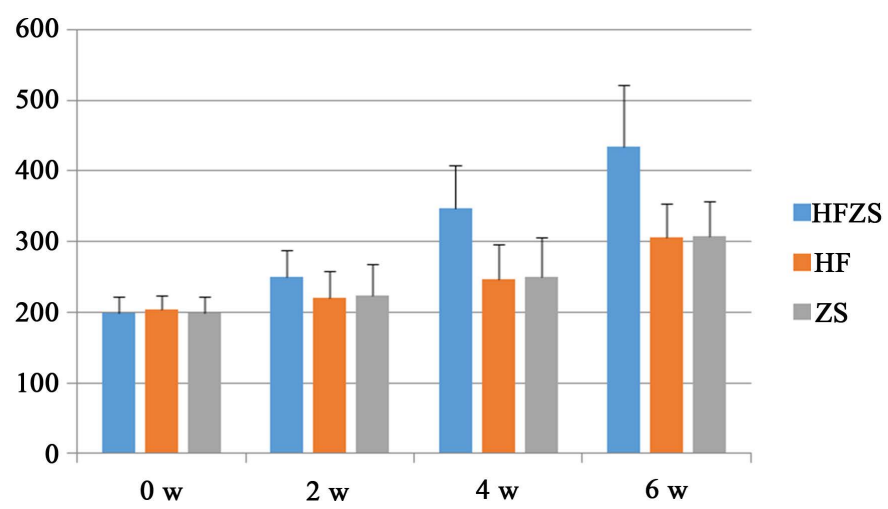

Figure 4. TGF- $\beta 1$ content in secretions among three groups.

Table 4. NO content in secretions among three groups (nmol/mg; $\bar{x} \pm s$ ).

\begin{tabular}{ccccc} 
Group & $n$ & $0 \mathrm{w}$ & $2 \mathrm{w}$ & $4 \mathrm{w}$ \\
\hline HFZS & 35 & $0.160 \pm 0.017$ & $0.188 \pm 0.017$ & $0.540 \pm 0.109^{* * \Delta \Delta}$ \\
HF & 32 & $0.161 \pm 0.017$ & $0.171 \pm 0.021$ & $0.438 \pm 0.111$ \\
ZS & 31 & $0.158 \pm 0.015$ & $0.158 \pm 0.016$ & $0.401 \pm 0.086$ \\
\hline
\end{tabular}

Notes: compare to HF group, ${ }^{*} P<0.05,{ }^{*}{ }^{*} P<0.01$; compare to ZS group, ${ }^{\triangle} P<0.05,{ }^{\triangle \triangle} P<0.01$. 


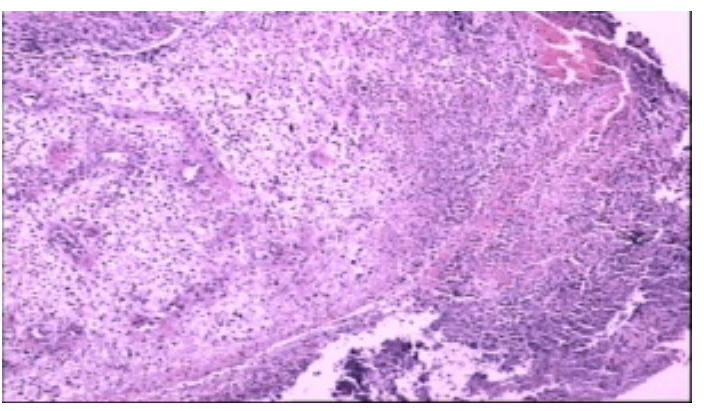

(a)

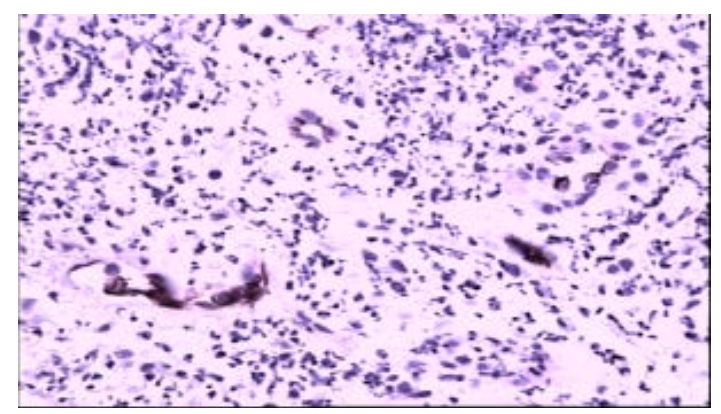

(c)

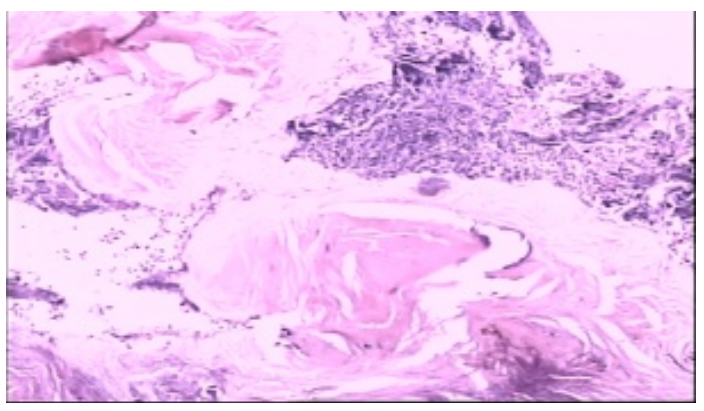

(b)

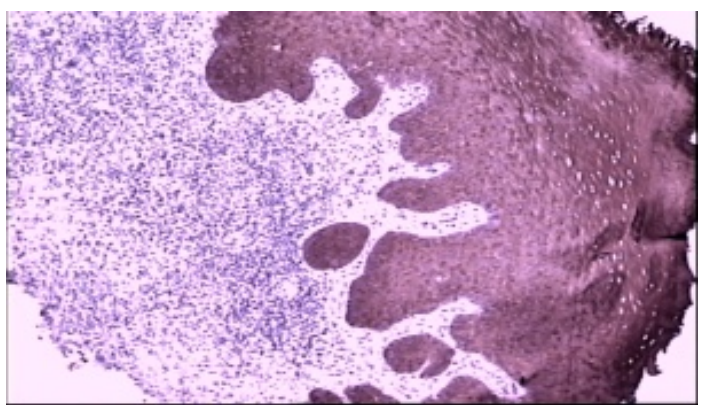

(d)

Figure 5. (a) HE staining, pink with no structure, mixed bacteria group and acute inflammatory exudate, neutrophils degeneration and necrosis (HE $\times 40$ ); (b) HE staining, inflammatory exudation of necrosis and inflammatory granulation tissue $(\mathrm{HE} \times 100)$; (c) Capillary endothelial cells positive expression $(\mathrm{CD} 34 \times 100)$; (d) Squamous epithelium completely positive expression $(\mathrm{CK} 19 \times 100)$.

Table 5. TGF- $\beta 1$ content in secretions among three groups (pg/mg, $\bar{x} \pm s$ ).

\begin{tabular}{|c|c|c|c|c|c|}
\hline Group & $n$ & $0 \mathrm{w}$ & $2 \mathrm{w}$ & $4 \mathrm{w}$ & $6 \mathrm{w}$ \\
\hline HFZS & 35 & $198.22 \pm 23.18$ & $249.53 \pm 37.72^{\triangle \Delta \Delta \Delta}$ & $347.28 \pm 60.92^{\Delta \Delta \Delta \Delta}$ & $435.30 \pm 87.25^{\triangle \Delta \Delta \Delta}$ \\
\hline $\mathrm{HF}$ & 32 & $203.92 \pm 19.86$ & $219.76 \pm 38.15$ & $246.41 \pm 49.31$ & $306.66 \pm 47.77$ \\
\hline $\mathrm{ZS}$ & 31 & $199.21 \pm 23.07$ & $223.71 \pm 44.23$ & $249.84 \pm 56.87$ & $307.59 \pm 49.45$ \\
\hline
\end{tabular}

Notes: compare to HF group, ${ }^{\Delta} P<0.05,{ }^{\triangle} P<0.01$; compare to ZS group, ${ }^{\wedge} P<0.05,{ }^{\wedge}{ }^{\star} P<0.01$.

\section{Conclusion}

Using the method of Huafu Zaisheng, the necrotic tendons and tissues could be liquefied as early as possible, and then promote the wound healing. Application of Huafuzaisheng method to deal with wounds, can remove necrotic tendons and fascia tissue as early as possible, can change the wound $\mathrm{pH}$ value and provide a variety of active factors (NO, TGF- $\beta 1$ ) and micro-environment for wound healing.

\section{Discussion}

Tendon infection and necrosis were common happened in patients with diabetes foot, they are difficult to treat, just a improper handling lead to amputation. They are associated with increased frequency and length of hospitalization and risk of lower extremity amputation [3].

Bromelain, a pineapple-derived enzyme mixture, is a widely used drug to improve tissue regeneration. Proteolytic, anti-bacterial, anti-inflammatory, and anti-oedematogenic effects account for this improvement on the systemic level [4]. Bromelain accounts for many therapeutic benefits like the treatment of angina pectoris, bronchitis, sinusitis, surgical trauma, and thrombophlebitis, debridement of wounds, and enhanced absorption of drugs, particularly antibiotics [5].

Nowadays, our clinical study shows that bromelain combined with Shengji corium elephatis mastic has good efficacy in diabetic foot wound repair. There is a close relationship between the $\mathrm{pH}$ value of ulcer microenviron- 
ment and wound repair. The research showed that diabetic foot ulcer wound exist a large number of necrosis of tendon and the organization, the $\mathrm{pH}$ value of the wound is weak alkaline. When using bromelain debridement to liquefied necrotic tendons, the $\mathrm{pH}$ value of wound secretion is acidic. When the wound necrosis tissue being remove and the granulation tissue begins to grow, the $\mathrm{pH}$ value of wound secretion tend to be neutral. Professor $\mathrm{Li}$ [6] pointed out that the acidic environment is conducive to the removal of necrotic tissue, the neutral environment is conducive to wound repair. The $\mathrm{pH}$ value of HFZS group change quickly, we think it is closely related to moisturizing effect of Shengji corium elephatis mastic. It is favor to the bromelain play the role of debridement.

Besides, nitric oxide plays an important role in the process of wound healing. Studies have shown that nitric oxide can promote tendon injury repair. After the injury, the increase of nitric oxide can ensure the blood supply of the injury area and prevent bacterial infection [7].

But, diabetic foot tendon injuries is a chronic process, mainly is refers to the tendon infection and necrosis. Observation shows that: in the treatment process, amount of Nitric oxide present a trend of increasing. We think that Nitric oxide play important role in degeneration tendon reversal and infection control. With the decrease of necrotic tissue, granulation tissue slowly wrapped the exposed tendons. We have not detected the reason and mechanism of the increase of nitric oxide.

However, a small amount of nitric oxide can contribute to the repair of damage, a large amount of Nitric oxide can make tendon degradation [8]. Bromelain debridement remove necrotic tissue may be associated with the role of a large number of Nitric oxide.

Transforming growth factor beta- 1 is a cytokine that plays multiple roles in wound healing but is also implicated in the pathogenesis of excessive scar formation [9]. TGF $\beta$ has shown to be active in almost all stages of tendon healing and has such varied effects as stimulating extrinsic cell migration, regulation of proteinases, fibronectin binding interactions, termination of cell proliferation via cyclin dependent kinase inhibitors and stimulation of collagen production. TGF $\beta-1$ mRNA expression has been shown to dramatically increase a short time after tendon injury and is thought, in particular, to play an important role in the initial inflammatory response [10].

But, what role can TGF $\beta$-1 play in the infection, necrosis of tendon repair? Our study shows that, when there is necrosis of the tendon, expression of TGF $\beta$ - 1 is the lowest. Along with the necrosis tendons reduce the expression of TGF $\beta$-1increase.We suspect that in the process of granulation tissue package tendons, TGF $\beta$ - 1 played an important role, promoting capillaries in growth tendon tissue, promoting moderate inflammatory response? Further studies are needed.

Huafu Zaisheng method using bromelain and Shengji corium elephatis mastic. Bromelain combined with Shengji corium elephatis mastic has good efficacy in diabetic foot wound repair. Shengji corium elephatis mastic improve the activity and bioavailability of bromelain. Shengji corium elephatis mastic provides nutrients for wound, including growth factors, the joint application, curative effect is obvious. Anyhow, Huafu Zaisheng method to remove necrotic tissue, change the wound $\mathrm{pH}$ value, provide a variety of active factors and microenvironment for wound healing. Still need to study its mechanism of action.

Huafu Zaisheng method is applicable to diabetic foot ulcers accompanied by necrosis of the tendon and the fascia. However, the application of the external treatment method must to keep wound drainage unobstructed. If the wound drainage not unobstructed, it can cause infection spread along the tendon to the proximal, even result in amputation. Ensure the wound drainage unobstructed throughout the diabetic foot treatment.

\section{References}

[1] Brem, H., Sheehan, P., Rosenberg, H.J., Schneider, J.S. and Boulton, A.J. (2006) Evidence-Based Protocol for Diabetic Foot Ulcers. Plastic and Reconstructive Surgery, 117, S193-S211. http://dx.doi.org/10.1097/01.prs.0000225459.93750.29

[2] Wagner, F.W. (1987) The Diabetic Foot. Orthopedics, 10, 163-172.

[3] Boulton, A.J., Vileikyte, L., Ragnarson-Tennvall, G. and Apelqvist, J. (2005) The Globalburden of Diabetic Foot Disease. Lancet, 366, 1719-1724. http://dx.doi.org/10.1016/S0140-6736(05)67698-2

[4] Aichele, K., Bubel, M., Deubel, G., Pohlemann, T. and Oberringer, M. (2013) Bromelain Down-Regulates Myofibroblast Differentiation in an in Vitro Wound Healing Assay. Naunyn-Schmiedeberg's Archives of Pharmacology, 386, 853-863. http://dx.doi.org/10.1007/s00210-013-0890-z

[5] Pavan, R., Jain, S., Shraddha, et al. (2012) Properties and Therapeutic Application of Bromelain: A Review. Biotech- 
nology Research International, 2012, Article ID: 976203. http://dx.doi.org/10.1155/2012/976203

[6] Li, J. (1999) Ulcer Disease Encyclopedia of China. Tianjin Science and Technology Publishing House, 34-35.

[7] Wang, R.J., Ghahary, A., Shen, Y.J., et al. (1997) Nitric Oxide Synthase Expression and Nitric Oxide Production Are Reduced in Hypertrophic Scar Tissue and Fibroblasts. Journal of Investigative Dermatology, 108, 438-444. http://dx.doi.org/10.1111/1523-1747.ep12289708

[8] Cho, N.S., Hwang, J.H., Lee, Y.T., et al. (2011) Tendinosis-Like Histologic and Molecular Changes of the Achilles Tendon to Repetitive Stress: A Pilot Study in Rats. Clinical Orthopaedics and Related Research, 469, 3172-3180. http://dx.doi.org/10.1007/s11999-011-2000-1

[9] Chang, J., Most, D., Stelnicki, E., et al. (1997) Gene Expression of Transforming Growth Factor Beta-1 in Rabbit Zone II Flexor Tendon Wound Healing: Evidence for Dual Mechanisms of Repair. Plastic \& Reconstructive Surgery, 100, 937-944. http://dx.doi.org/10.1097/00006534-199709001-00016

[10] Molloy, T., Wang, Y. and George, A.C. (2003) The Roles of Growth Factors in Tendon and Ligament Healing. Sports Medicine, 33, 381-394. http://dx.doi.org/10.2165/00007256-200333050-00004 